\title{
Elastic Fracture Toughness for Ductile Metal Pipes with Circumferential Surface Cracks
}

\author{
Chun-Qing $\mathrm{Li}^{1, \mathrm{a}^{*}}$, Guoyang $\mathrm{Fu}^{1, \mathrm{~b}}$ and Shangtong Yang ${ }^{2, \mathrm{c}}$ \\ ${ }^{1}$ School of Engineering, RMIT University, GPO Box 2476, Melbourne 3001, Australia \\ ${ }^{2}$ Department of Civil and Environmental Engineering, University of Strathclyde, Glasgow G1 1XJ, UK \\ achunqing.li@rmit.edu.au, bguoyang.fu@rmit.edu.au, cshangtong.yang@strath.ac.uk
}

Keywords: Elastic fracture toughness, Circumferential crack, Plasticity, Stress intensity factor, Ductile Metal pipes.

\begin{abstract}
Surface cracks have long been recognized as a major cause for potential failures of metal pipes. In fracture analysis, the widely used method is based on linear elastic fracture mechanics. However, for ductile metal pipes, it has been known that the existence of plasticity results in easing of stress concentration at the crack front. This will ultimately increase the total fracture toughness. Therefore, when using linear elastic fracture mechanics to predict fracture failure of ductile metal pipes, the plastic portion of fracture toughness should be excluded. Otherwise, the value of fracture toughness will be overestimated, resulting in an under-estimated probability of failure. This paper intends to derive a model of elastic fracture toughness for steel pipes with a circumferential crack. The derived elastic fracture toughness is a function of crack geometry and material properties of the cracked pipe. The significance of the derived model is that the well-established linear elastic fracture mechanics can be used for ductile materials in predicting the fracture failure.
\end{abstract}

\section{Introduction}

Pipelines are essential infrastructure that play a pivotal role in a nation's economy, prosperity, health, environment, social well-being and quality of life. Various materials have been used to make pipes, a significant portion of which are made of ductile metal materials, e.g. steel, with a range of grades. Due to long term service and exposure to corrosive environment, aging and deterioration of metal pipes have resulted in failures way before the end of intended design life.

Through investigation, it has been found that most metal pipe failures are of fracture type, caused by the propagation of a surface crack or defect. For cracked ductile metal pipes, the crack front yields before the stress intensity factor reaches its critical value and the yielding eases the stress concentration at the crack front. As a result, plastic yielding increases the fracture resistance. In order to enable the use of linear elastic fracture mechanics (LEFM) for ductile materials, the plasticity induced portion should be excluded from the overall fracture toughness. Otherwise the value of fracture toughness will be overestimated.

Extensive research has been conducted on calculating the stress intensity factors for surface cracks in pipes. However, stress intensity factors are only applicable for elastic materials and plastic materials under small scale yielding conditions [1]. For ductile materials with considerable development of plastic deformation, $J$ integral or Crack Tip Opening Displacement has to be used and non-linear finite element analyses are often resorted to for investigating the behavior of cracked pipes, which is quite complicated with high computational demand. If the portion of the fracture resistance within the elastic range of the ductile material can be determined, the widely available results of linear elastic fracture mechanics can be readily applied. Yang et al. [2] proposed an analytical model of elastic fracture toughness for steel pipes, but this study is limited to internal longitudinal surface cracks under internal pressure only.

This paper aims to develop a model of the elastic fracture toughness for circumferentially cracked pipes under single loading, i.e., axial tension and bending. The elastic fracture toughness in the developed model is a function of geometry and material properties of the cracked pipe. After indirect verification of the proposed model, parametric studies are conducted to investigate the effect of the 
key parameters on the failure modes and elastic fracture toughness. The outcome from this study will allow the use of extensive results based on LFEM by engineers and asset managers for both design and assessment of ductile metal pipes, which can prevent further failures of pipes.

\section{Failure Criteria for Cracked Ductile Metal Pipes}

It is known that a cracked brittle pipe fails when the stress intensity factor $K_{I}$ at the crack front exceeds the fracture toughness $K_{I C}$. For pipes made of ductile materials, the fracture toughness is increased due to the existance of plasticity. To enable the use of LEFM for ductile materials, a term called elastic fracture toughness, can be proposed and derived by excluding the plasticity induced portion from the overall fracture toughness. Consequently, the following failure criteria can be used to assess the fracture conditions of ductile pipes

$$
K_{I} \leq K_{I C}^{e} \text {. }
$$

where, $K_{I C}^{e}$ is the proposed elastic fracture toughness.

For ductile metal pipes, failures often occur due to the interaction between brittle fracture and plastic collapse. Two parameters have been employed to quantify the two failure modes separately in the structural integrity assessment of cracked pipes as follows [3]

$$
\begin{aligned}
& K_{r}=K_{I} / K_{I C} . \\
& L_{r}=P / P_{L} .
\end{aligned}
$$

where, $P$ is the applied loading and $P_{L}$ is the corresponding plastic load limit of the cracked pipes.

Based on experiments, a relationship between $K_{r}$ and $L_{r}$ has been developed as follows [4]

$$
K_{r}=\left(1+0.5 L_{r}^{2}\right)^{-0.5}\left[0.3+0.7 \exp \left(-0.65 L_{r}^{6}\right)\right] \text {. }
$$

where, the maximum value of $L_{r}$ is defined as $\bar{\sigma} / \sigma_{y}, \sigma_{y}$ is the yield stress and $\bar{\sigma}$ is the uniaxial flow stress, calculated as the average of the yield and ultimate tensile strengths.

\section{Elastic Fracture Toughness for Circumferential Cracks in Pipes}

Stress Intensity Factor (SIF) Equations. The axial tension and bending forces only result in the opening mode (Mode I) for a circumferential surface crack in pipes (Fig. 1). The Mode I stress intensity factors $K_{I}$ for any point along the crack front can be expressed as follows

$$
K_{I}=\sigma \sqrt{\pi a / Q} F_{I}\left(a / d, a / c, d / R_{i}, \varphi\right) .
$$

where, $\sigma$ can be the applied stress, $a$ is the crack depth, $Q$ is the shape factor for an ellipse, $d$ is the pipe thickness, $c$ is half of crack length, $R_{i}$ is the pipe internal radius, $\varphi$ is used to define the position along the semi-elliptical crack, and $F_{I}$ is the influence coefficient for Mode I.

The results of stress intensity factors for circumferential cracks in table form from Raju and Newman [5] are used. In addition, three-dimensional Finite Element Analyses using the modelling technique in Li et al. [6] are performed to obtain the stress intensity factors for cracks with aspect ratios $0.2,0.4$ and 1.5. Based on all the above results, equations of the influence coefficients at the deepest and surface points of the crack front for pipes under axial tension and bending respectively are obtained by performing non-linear regression as follows

$$
\begin{aligned}
& F_{I}=\left\{h_{1}+h_{2}(a / c)+\left[h_{3}+h_{4}(a / c)+h_{5}(a / c)^{2}\right](a / d)^{2}+\left[h_{6}+h_{7}(a / c)+h_{8}(a / c)^{2}\right]\right. \\
& \left.(a / d)^{4}\right\} \exp \left(h_{9}\left(d / R_{i}\right)\right)
\end{aligned}
$$

where, the values of coefficient $h_{i}(i=1,2, \cdots, 9)$ are listed in Table $1, a / c$ ranges from $0.4-1.5$, $a / d$ ranges from $0.2-0.8$, and $d / R_{i}$ ranges from $0.1-1$. The Eq. (6) is within $\mp 6 \%$ of the finite element results.

For pipes under axial tension or bending, the stress intensity factors can be expressed as follows

$$
K_{I}(N)=\sigma_{a} \sqrt{\pi a / Q} F_{I}(N)
$$




$$
K_{I}(M)=\sigma_{b} \sqrt{\pi a / Q} F_{I}(M)
$$

where, $K_{I}(N)$ and $K_{I}(M)$ are the stress intensity factors, while $F_{I}(N)$ and $F_{I}(M)$ are the influence coefficients, for pipes under axial tension $N$ and bending $M$ respectively.
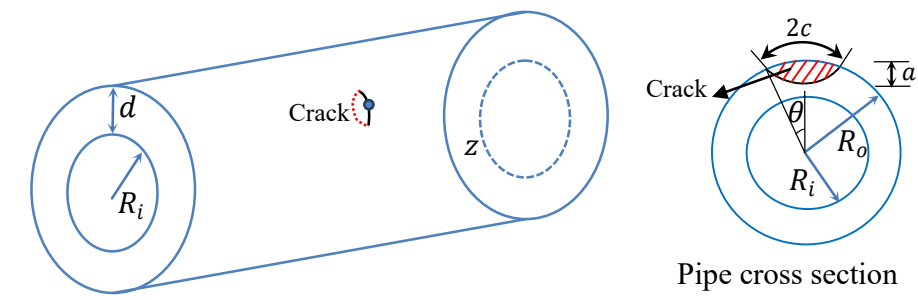

Pipe cross section
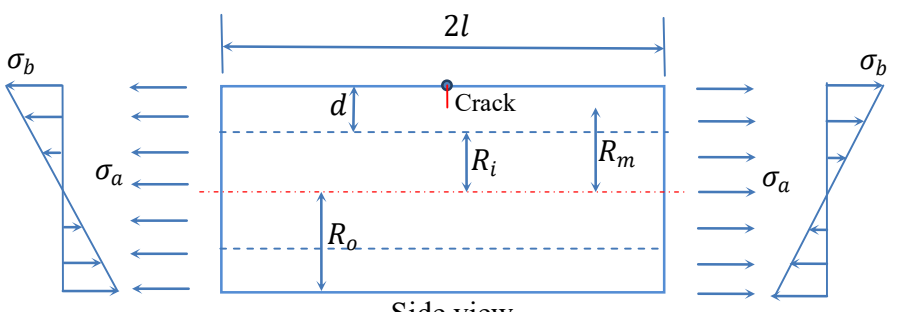

Fig. 1 A pipe with a circumferential crack subjected to axial tension and bending.

Table 1. Values of coefficient $h_{i}$ in Eq. (6).

\begin{tabular}{ccccc}
\hline Coefficients & \multicolumn{2}{c}{ Under tension } & \multicolumn{2}{c}{ Under bending } \\
\cline { 2 - 5 } & Deepest point & Surface point & Deepest point & Surface point \\
$\boldsymbol{h}_{\mathbf{1}}$ & 1.086 & 0.550 & 0.999 & 0.566 \\
$\boldsymbol{h}_{\mathbf{2}}$ & -0.052 & 0.568 & -0.036 & 0.530 \\
$\boldsymbol{h}_{\mathbf{3}}$ & 2.168 & -0.545 & 2.827 & -0.038 \\
$\boldsymbol{h}_{\mathbf{4}}$ & -3.898 & 2.518 & -5.938 & 0.724 \\
$\boldsymbol{h}_{\mathbf{5}}$ & 1.680 & -1.325 & 2.768 & -0.290 \\
$\boldsymbol{h}_{\mathbf{6}}$ & -1.778 & 0.942 & -2.460 & 0.362 \\
$\boldsymbol{h}_{\mathbf{7}}$ & 3.667 & -2.355 & 5.382 & -0.600 \\
$\boldsymbol{h}_{\mathbf{8}}$ & -1.708 & 1.183 & -2.615 & 0.143 \\
$\boldsymbol{h}_{\mathbf{9}}$ & 0.046 & 0.021 & 0.109 & 0.059 \\
\hline
\end{tabular}

Plastic Limit Loads. In this study, the plastic load limit solutions for pipe under axial tension and bending, derived by Kim et al. [7] based on finite element limit analyses, are employed as follows The plastic limit load solution for axial tension $N_{L}$ is given by

$$
N_{L}=2 \pi \sigma_{y} R_{i} d\left[1+A_{1}\left(\frac{a}{d}\right)+A_{2}\left(\frac{a}{d}\right)^{2}\right]
$$

where, $A_{1}=0.066-0.038\left(\frac{\theta}{\pi}\right)-0.960\left(\frac{\theta}{\pi}\right)^{2}, A_{2}=-0.060-1.525\left(\frac{\theta}{\pi}\right)+1.427\left(\frac{\theta}{\pi}\right)^{2}, \theta=\frac{c}{R_{m}}$ and $R_{m}$ is the mean radius of the pipe.

$M_{L}$ is the plastic limit load solution for bending and given by

$$
M_{L}=4 \sigma_{y} R_{i}^{2} d\left[1+B_{1}\left(\frac{a}{d}\right)+B_{2}\left(\frac{a}{d}\right)^{2}\right]
$$

where, $B_{1}=0.074-0.169\left(\frac{\theta}{\pi}\right)$, and $B_{2}=-0.086-1.013\left(\frac{\theta}{\pi}\right)$.

Derivation of Elastic Fracture Toughness. For pipes with circumferential cracks under either axial tension $N$ or bending $M$, the uniform axial stress $\sigma_{a}$ and maximum bending stress $\sigma_{b}$ can be represented as follows

$$
\sigma_{a}=\frac{N}{\pi\left(R_{o}^{2}-R_{i}^{2}\right)}
$$




$$
\sigma_{b}=\frac{4 M R_{o}}{\pi\left(R_{o}^{4}-R_{i}^{4}\right)}
$$

where, $R_{o}$ is the external radius of the pipe.

For pipes with circumferential cracks under axial tension, substitute Eqs. (7), (9) and (11), and Eqs. (2) and (3) become

$$
\begin{aligned}
& K_{r}=\frac{K_{I}}{K_{I C}}=\frac{\sigma_{a} \sqrt{\pi a / Q} F_{I}(N)}{K_{I C}} \\
& L_{r}=\frac{N}{N_{L}}=\frac{\pi \sigma_{a}\left(R_{o}^{2}-R_{i}^{2}\right)}{2 \pi \sigma_{y} R_{i} d\left[1+A_{1}\left(\frac{a}{d}\right)+A_{2}\left(\frac{a}{d}\right)^{2}\right]}
\end{aligned}
$$

By dividing Eq. (13) by Eq. (14), the following expression can be obtained

$$
L_{r}=\frac{\sqrt{Q} K_{r} K_{I C}\left(R_{o}^{2}-R_{i}^{2}\right)}{\sqrt{\pi a} F_{I}(N)\left\{2 \sigma_{y} R_{i} d\left[1+A_{1}\left(\frac{a}{d}\right)+A_{2}\left(\frac{a}{d}\right)^{2}\right]\right\}}
$$

Solving Eqs. (4) and (15) simultaneously, the critical value $K_{r c}$ of $K_{r}$ at fracture can be derived as follows

$$
\begin{aligned}
& K_{r c}=\left(1+0.5\left(\frac{\sqrt{Q} K_{r c} K_{I C}\left(R_{o}^{2}-R_{i}^{2}\right)}{\sqrt{\pi a} F_{I}(N)\left\{2 \sigma_{y} R_{i} d\left[1+A_{1}\left(\frac{a}{d}\right)+A_{2}\left(\frac{a}{d}\right)^{2}\right]\right\}}\right)^{2}\right)^{-0.5} \\
& {\left[0.3+0.7 \exp \left(-0.65\left(\frac{\sqrt{Q} K_{r c} K_{I C}\left(R_{o}^{2}-R_{i}^{2}\right)}{\sqrt{\pi a} F_{I}(N)\left\{2 \sigma_{y} R_{i} d\left[1+A_{1}\left(\frac{a}{d}\right)+A_{2}\left(\frac{a}{d}\right)^{2}\right]\right\}}\right)^{6}\right)\right]}
\end{aligned}
$$

Similarly, for pipes with circumferential cracks under bending, the relationship between $K_{r}$ and $L_{r}$ can be expressed as follows

$$
L_{r}=\frac{\sqrt{\pi Q} K_{r} K_{I C}\left(R_{o}^{4}-R_{i}^{4}\right)}{16 \sqrt{a} F_{I}(M) R_{o}\left\{\sigma_{y} R_{i}^{2} d\left[1+B_{1}\left(\frac{a}{d}\right)+B_{2}\left(\frac{a}{d}\right)^{2}\right]\right\}}
$$

Solving Eqs. (4) and (17) simultaneously, the critical value $K_{r c}$ of $K_{r}$ for cracked pipes under bending at fracture can be derived as follows

$$
\begin{aligned}
& K_{r c}=\left(1+0.5\left(\frac{\sqrt{\pi Q} K_{r c} K_{I C}\left(R_{o}^{4}-R_{i}^{4}\right)}{16 \sqrt{a} F_{I}(M) R_{o}\left\{\sigma_{y} R_{i}^{2} d\left[1+B_{1}\left(\frac{a}{d}\right)+B_{2}\left(\frac{a}{d}\right)^{2}\right]\right\}}\right)^{2}\right)^{-0.5} \\
& {\left[0.3+0.7 \exp \left(-0.65\left(\frac{\sqrt{\pi Q} K_{r c} K_{I C}\left(R_{o}^{4}-R_{i}^{4}\right)}{16 \sqrt{a} F_{I}(M) R_{o}\left\{\sigma_{y} R_{i}^{2} d\left[1+B_{1}\left(\frac{a}{d}\right)+B_{2}\left(\frac{a}{d}\right)^{2}\right]\right\}}\right)^{6}\right)\right]}
\end{aligned}
$$

where, $\left\{\left(1+0.5\left(\frac{\bar{\sigma}}{\sigma_{y}}\right)^{2}\right)^{-0.5}\left[0.3+0.7 \exp \left(-0.65\left(\frac{\bar{\sigma}}{\sigma_{y}}\right)^{6}\right)\right]\right\} \leq K_{r c} \leq 1$.

When the critical state of pipe failure is reached, the stress intensity factor $K_{I}$ for the brittle fracture will become the elastic critical limit, i.e., elastic fracture toughness $K_{I C}^{e}$. It can be expressed as follows

$$
K_{I C}^{e}=K_{r c} K_{I C}
$$

From Eqs. (16), (18) and (19), it can be seen that $K_{I C}^{e}$ is a function of the pipe and crack geometry, material properties. 


\section{Verification and Discussions}

To verify the derived elastic fracture toughness, ideally experimental results should be employed to do the comparison. However, from literature review, it has been found to be extremely difficult. Therefore, the proposed model is verified indirectly in this paper. The $K_{r}$ calculated from the proposed model are compared with these from the literature. Miller [8] and Staat and $\mathrm{Vu}$ [9] summarized the burst test results of pipes with circumferential cracks. Most cracks considered have very low aspect ratios, which are out of the applicable range of Eqs. (7) and (8). For some tests, no specific material property data were documented. Schulze et al. [10] carried out tests on steel pipes with artificially and fatigue induced circumferential cracks of various lengths. The $K_{r}$ of the failed cracked pipes is calculated from the proposed model (Eq. 15 or 17) and the ASME boiler and Pressure code [11], which was often used to interpret the experimental results of cracked pipes, e.g. [12]. From Table 2, it can be seen that satisfactory agreement has been achieved. In addition, the plastic load limit has been verified by comparison with analytical solutions developed based on equilibrium stress fields [7] while Eq. (4) has been derived as a low bound of the failure assessment diagrams obtained based on the reference stress approach [4], which will provide some safety margin for assessment.

Table 2. Comparison of $K_{r}$ for the deepest point between $\mathrm{Eq}(17)$ and those from [11].

\begin{tabular}{ccccccccc}
\hline $\boldsymbol{R}_{\boldsymbol{i}}[\mathrm{mm}]$ & $\boldsymbol{a}[\mathbf{m m}]$ & $\frac{\boldsymbol{a}}{\boldsymbol{c}}$ & $\frac{\boldsymbol{a}}{\boldsymbol{d}}$ & $\boldsymbol{K}_{\boldsymbol{I C}}[\mathbf{M P a} / \sqrt{\boldsymbol{m}}]$ & $\mathbf{M}[\mathbf{k N m}]$ & $\boldsymbol{K}_{\boldsymbol{r}}[\mathbf{1 0}]$ & $\boldsymbol{K}_{\boldsymbol{r}}$ (Proposed model) & Error [\%] \\
\hline 33.5 & 3 & 0.25 & 0.75 & 98 & 9.1 & 0.84 & 0.80 & 5 \\
\hline
\end{tabular}

Using the proposed model, the structural integrity of pipes with circumferential external surface cracks under tension and bending can be assessed by Eq. (1), in which $K_{I}$ is calculated by Eqs. (7) and (8) for axial tension and bending respectively while and $K_{I C}^{e}$ is determined by Eq. (19).

As the elastic fracture toughness $K_{I C}^{e}$ is a function of the crack and pipe geometry, and material properties, the developed model can be applied to study the effects of the key parameters. The $K_{r}-L_{r}$ curve can be produced by Eq. (4), with the maximum value of $L_{r}$ limited by yield strength and ultimate strength. From Eqs. (15) and (17), it can be seen that with the increase of the applied loading, either axial tension or bending, $K_{r}$ linearly increases with $L_{r}$ for given crack and pipe geometry, and material properties. The slopes of the $K_{r}-L_{r}$ lines are dependent on the geometrical and material properties but independent of the applied loading.

Figs. 2 and 3 demonstrate the effect of material properties on failure mode for the deepest point and surface point for a given cracked pipe. The same trend of the effects has been found for bending and axial tension as shown in Figs 2 and 3. For given pipe and crack geometry, the larger the yield strength of the pipe material is, the higher portion of the brittle fracture the pipe will experience, which indicates that the pipe will fail in a more brittle way. Similarly, the larger the fracture toughness is, the higher portion of plastic deformation the pipe will endure, which means the pipe will fail in a more plastic manner. This makes sense as the larger the yield strength (fracture toughness) is, the higher capacity of the material to withstand plastic deformation (brittle fracture) development. Therefore, the pipes tend to fail in a brittle (ductile) manner.

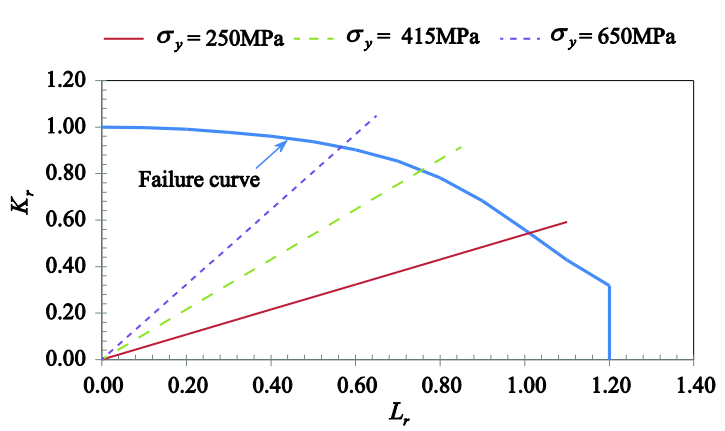

a) Deepest point



b) Surface point

Fig. 2 Effect of yield strength on pipe failure. 

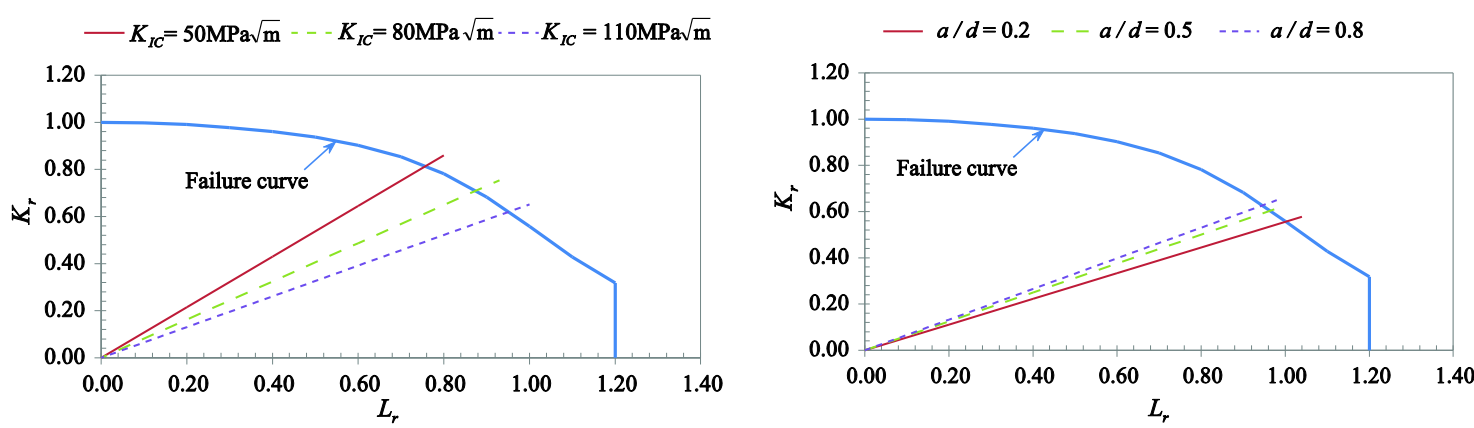

Fig. 3 Effect of fracture toughness on pipe failure. Fig. 4 Effect of $a / d$ on pipe failure.

The effect of relative crack depth $a / d$ has also been investigated. As shown in Fig. 4, when the relative depth increases from $0.2-0.8$, the pipe material demonstrates more brittleness. This can be explained by the amount of materials ahead of the crack front. Larger relative depth means less material to develop plastic deformation. Therefore, the pipe failure will tend to be at a lower level of plasticity.

\section{Conclusions}

A model of elastic fracture toughness for ductile metal pipes with circumferential external surface cracks under axial tension and bending has been developed. A detailed examination of the developed model reveals that the elastic fracture toughness is a function of the geometry and material properties of the cracked pipe, but independent of the applied single loading, i.e., axial tension or bending in the present study. It has been found that increasing the fracture toughness and yield strength will result in the pipe failure in a more ductile and brittle way respectively. In addition, it is found that an increase in the relative crack depth leads to less plastic deformation development in the pipe before failure. It is concluded that with the proposed model, ductile metal pipes with circumferentially cracks can be assessed more accurately.

\section{Acknowledgements}

Financial support from Australian Research Council under DP140101547 and LP150100413 is gratefully acknowledged.

\section{References}

[1] T. L. Anderson, Fracture mechanics: Fundamentals and Applications, CRC Press, Boca Raton, Florida, 1991.

[2] S. T. Yang, C. Q. Li, W. Yang, Analytical model of elastic fracture toughness for steel pipes with internal cracks, Eng. Fract. Mech. 153 (2016) 50-60.

[3] I. Milne, R. A. Ainsworth, A. R. Dowling, A. T. Stewart, Background to and Validation of CEGB Report R/H/R6-Revision 3, Int. J. Pres. Ves. Pip 32(1-4) (1988) 105-196.

[4] SINTAP, Structural Integrity Assessment Procedures for European Industry-Final Procedure, European Union Project No.BE95-1426, 1999.

[5] I. S. Raju, J. C. Newman, Stress intensity factors for circumferential surface cracks in pipes and rods, Fracture Mechanics: Seventeenth Volume, ASTM STP (Third Edn), 905 (1986) 789-805.

[6] C. Q. Li, G. Y. Fu, W. Yang, Stress intensity factors for inclined external surface cracks in pressurized pipes, submitted to Engineering Fracture Mechanics (2016). 
[7] Y. J. Kim, D. J. Shim, N. S. Huh, Y. J. Kim, Finite element based plastic limit loads for cylinders with part-through surface cracks under combined loading, Int. J. Pres. Ves. Pip 80(7-8) (2003) 527-540.

[8] A. G. Miller, Review of test results for ductile failure pressure of cracked spherical and cylindrical pressure vessels, UK: CEGB; 1984.

[9] M. Staat, D. K. Vu, Limit analysis of flaws in pressurized pipes and cylindrical vessels, Part II: Circumferential defects, Eng Fract Mech, 97 (2013) 314-333.

[10]H. D. Schulze, G. Togler, E. Bodmann, Fracture mechanics analysis on the initiation and propagation of circumferential and longitudinal cracks in straight pipes and pipe bends, Nucl. Eng. Des. 58(1) (1980) 19-31.

[11]ASME Boiler and Pressure Vessel Code, section 11, In service inspection of nuclear power plant components, 1974, Article A. 3000, p. 117.

[12]K. R. Brown, B. Zybenko, The fracture mechanics of alluminum alloy gas cylinders, Eng. Fract. Mech. 15(1-2) (1981) 1-20. 